\title{
Correction to: A Hybrid Biofuel and Triboelectric Nanogenerator for Bioenergy Harvesting
}

Cite as

Nano-Micro Lett.

(2020) 12:92

Published online: 16 April 2020

(C) The Author(s) 2020

\author{
Hu Li ${ }^{1,2}$, Xiao Zhang ${ }^{1,2}$, Luming Zhao ${ }^{2,3}$, Dongjie Jiang ${ }^{2,3}$, Lingling $\mathrm{Xu}^{2,3}$, Zhuo Liu ${ }^{1,2}$, \\ Yuxiang $\mathrm{Wu}^{4}$, Kuan $\mathrm{Hu}^{6}$, Ming-Rong Zhang ${ }^{6}$, Jiangxue Wang ${ }^{1}$, Yubo Fan ${ }^{1}$, \\ Zhou $\mathrm{Li}^{2,3,5} \bowtie$ \\ The original article can be found online at https://doi.org/10.1007/s40820-020-0376-8. \\ $\triangle$ Jiangxue Wang, wangjiangxue@buaa.edu.cn; Yubo Fan, yubofan@buaa.edu.cn; Zhou Li, zli@binn.cas.cn \\ 1 Beijing Advanced Innovation Centre for Biomedical Engineering, Key Laboratory for Biomechanics \\ and Mechanobiology of Chinese Education Ministry, School of Biological Science and Medical \\ Engineering, Beihang University, Beijing 100083, People's Republic of China \\ 2 CAS Center for Excellence in Nanoscience, Beijing Key Laboratory of Micro-Nano Energy and Sensor, \\ Beijing Institute of Nanoenergy and Nanosystems, Chinese Academy of Sciences, Beijing 100083, \\ People's Republic of China \\ 3 School of Nanoscience and Technology, University of Chinese Academy of Sciences, Beijing 100049, \\ People's Republic of China \\ 4 School of Physical Education, Jianghan University, Wuhan 430056, People's Republic of China \\ 5 Center on Nanoenergy Research, School of Physical Science and Technology, Guangxi University, \\ Nanning 530004, People's Republic of China \\ 6 Department of Radiopharmaceuticals Development, National Institute of Radiological Sciences, National \\ Institutes for Quantum and Radiological Science and Technology, Chiba 263-8555, Japan
}

\section{Correction to: Nano-Micro Lett. (2020) 12:50 https://doi.org/10.1007/s40820-020-0376-8}

In the original publication, the authors' contribution is missing in the acknowledgment section. The correct acknowledgement is provided in this correction. Also, in Fig. 4, the second (c) after figure (d) should be read as (e). In Fig. 5 (i), the Y-axis label "Current $(\mu \mathrm{A})$ " should be read as "Voltage".

Acknowledgments The authors thank the support of National Key R\&D Project from Minister of Science and Technology, China (2016YFA0202703), National Natural Science Foundation of China (Nos. 61875015, 31571006, 81601629, 21801019 and 11421202), the 111 Project (Project No.: B13003), the Beijing Natural Science Foundation (2182091), Wuhan Municipal Science and Technology Bureau (Grant No. 2017060201010166), and the National Youth Talent Support Program. Author contributions: H. $\mathrm{Li}, \mathrm{X}$. Zhang contributed equally to this work. Prof. Z. Li and H. Li designed this experiment. $\mathrm{H}$. Li and X. Zhang carried out the experiment. H. Li wrote the paper. L.M. Zhao, D.J. Jiang, L.L.
Xu, Z. Liu, Y.X. Wu, K. Hu and M.R. Zhang assisted in drawing the diagram and characterized the materials. Prof. J.X. Wang, Prof. Y.B. Fan and Prof. Z. Li supervised the project. All authors reviewed and commented on the manuscript.

Open Access This article is licensed under a Creative Commons Attribution 4.0 International License, which permits use, sharing, adaptation, distribution and reproduction in any medium or format, as long as you give appropriate credit to the original author(s) and the source, provide a link to the Creative Commons licence, and indicate if changes were made. The images or other third party material in this article are included in the article's Creative Commons licence, unless indicated otherwise in a credit line to the material. If material is not included in the article's Creative Commons licence and your intended use is not permitted by statutory regulation or exceeds the permitted use, you will need to obtain permission directly from the copyright holder. To view a copy of this licence, visit http://creativecommons.org/licenses/by/4.0/. 